\title{
Prediction Method of Deep Horizontal Displacement of Slope Soil Based on Damped Holt-Winters Model
}

\author{
Kai Yan1, Jiaye $\mathbf{W u}^{2 *}$, Yingqian Zhang², Lu Yang3, Yixiong Zhang2, Haiyang Yuan4, Ying Huang4 \\ ${ }^{1}$ School of Management of Sichuan University of Science \& Engineering, Zigong, China \\ ${ }^{2}$ Sichuan Shengtuo Testing Technology Co. LTD., Zigong, China \\ ${ }^{3}$ School of Civil Engineering of Northeast Forestry University, Harbin, China \\ ${ }^{4}$ School of Civil Engineering of Chengdu University of Technology, Chengdu, China \\ Email:1411725547@qq.com, *wujy@scentralit.com
}

How to cite this paper: Yan, K., Wu, J.Y., Zhang, Y.Q., Yang, L., Zhang, Y.X., Yuan, H.Y. and Huang, Y. (2019) Prediction Method of Deep Horizontal Displacement of Slope Soil Based on Damped Holt-Winters Model. Journal of Service Science and Management, 12, 391-406.

https://doi.org/10.4236/jssm.2019.123027

Received: February 21, 2019

Accepted: April 12, 2019

Published: April 15, 2019

Copyright $\odot 2019$ by author(s) and Scientific Research Publishing Inc. This work is licensed under the Creative Commons Attribution International License (CC BY 4.0).

http://creativecommons.org/licenses/by/4.0/

\begin{abstract}
The prediction of deep horizontal displacement of slope soil is an important part of slope deformation monitoring, which has important guiding significance for the prevention of slope safety accidents. Holt-Winters model is suitable to predict the data series of deep horizontal displacement of slope soil, which show both trend growth and seasonal fluctuation. Firstly, this paper selected the data set as the original data for empirical analysis which is deep horizontal displacement of soil after pretreatment from the specific slope monitoring project, then used the Holt-winters' damped model to perform data mining, finally, compared with the traditional prediction methods including the neural-network model and the k-nearest neighbor classification. The results show that the damped Holt-winters model has the highest prediction accuracy.
\end{abstract}

\section{Keywords}

Safety Engineering, Deep Horizontal Displacement of Slope Soil, Damped Holt-Winters Model, Forecasting

\section{Introduction}

Slope stability has always been an important research content of slope engineering [1]. In recent years, with the rapid development of China's social economy, large scale rock mass engineering has also been rapid growth. But mountain road, railway and other engineering constructions had formed a large number of slopes, these slopes once due to natural or manmade damage caused by slope in- 
stability, will lead to serious disasters and accidents. Now, landslides have become one of the three major geological disasters in the world along with earthquakes and volcanoes [2] [3], and there are no shortage of painful lessons in this regard, both at home and abroad [4]. For example, in 1963, more than 2500 people died in the landslide on the slope of the Wayon reservoir in Italy. In 1970, a 7.7 magnitude earthquake struck about $25 \mathrm{~km}$ west of the city of Chimbote, Peru, triggering massive landslides that killed 67,000 people. In June 2014, a landslide occurred between Lianghekou and Zhenzi Street stations of the Chongqing railway Qian, causing the detour or suspension of 29 passengers trains. In November 2014, a landslide occurred on the Xunyang to Chongxi up-bound line of Xiangyu railway in Shaanxi province, causing the derailment of $1-12$ carriages of the 75228 train, resulting in the death of two drivers and the delay of some trains.

It can be seen that no matter the collapse and landslide of natural slope, or the instability of artificial slope caused by human engineering activities, these geological disasters have caused huge losses to economic construction and people's property [5]. Therefore, it is of great theoretical significance and practical value to explore methods to prevent slope accidents.

However, slope engineering is an uncertain and nonlinear complex system [6], and there are a variety of slope safety-monitoring technologies at home and abroad. For example, In 2010, Pieraccini, Barla, Gischig et al. firstly used GB-In SAR to monitor and study the deformation of slope [7] [8] [9]. In 2012, Ruan Zhixin used the inclinometer to monitor the expressway slope and proved the correctness of the monitoring [10]. In 2013, Wang Zhiyong used In SAR technology to monitor the landslide in Beijing's Fangshan area [11]. In 2015, Xu Maolin used TM30 to conduct a monitoring experiment on the slope displacement of an open pit mine in Anshan city, realizing automatic monitoring [12], etc. Most of these slope engineering monitoring technologies are used to monitor slope deformation, so it is concluded that slope deformation is of great importance to slope stability analysis. Therefore, this paper attempts to start with the deep horizontal displacement of soil in slope deformation monitoring, and make a prediction and empirical analysis of the deep horizontal displacement of soil using the Holt-Winters' damped model based on the WEKA data mining platform.

\section{Building Holt-Winters Model}

\subsection{Problem Description}

In slope deformation monitoring, the value of deep horizontal displacement of soil is a non-stationary time series that changes with seasonal cycles and has a certain increasing (or decreasing) trend. The traditional prediction methods of stationary time series, such as neural-network model and IBK model of the k-nearest neighbor classification, are not ideal for the prediction results of this kind of value [13]. However, the Holt-winters prediction model is suitable for the prediction of time series problems with seasonal fluctuations and trend growth (or decrease), because it can deal with both trend and seasonal changes 
[14] and properly filter the influence of random fluctuations.

\subsection{Building Model}

The basic idea of Holt-winters model is to analyze and study the time series with linear trend, seasonal variation and random fluctuation, and combine them with exponential smoothing method to estimate the long-term trend, trend increment and seasonal fluctuation respectively, and then establish a prediction model to extrapolate the predicted value [15]. Its seasonal model includes addition model and multiplication model.

The predictive extrapolation formula of Holt-winters' multiplication model is:

$$
y_{t+h}^{\prime}=\left(a_{t}+h b_{t}\right) s_{t+h-m}
$$

The calculation formulas of $a, b$ and $s$ are as follows:

$$
\begin{gathered}
a_{t}=\alpha\left(y_{t} / s_{t-m}\right)+(1-\alpha)\left(a_{t-1}+b_{t-1}\right) \\
b_{t}=\beta\left(a_{t}-a_{t-1}\right)+(1-\beta) b_{t-1} \\
s_{t}=\gamma\left[y_{t} /\left(a_{t-1}-b_{t-1}\right)\right]+(1-\gamma) s_{t-m}
\end{gathered}
$$

In order to predict a smaller time period data sets, on the basis of the multiplication model, add extension coefficient $\phi$; Furthermore, the predictive extrapolation formula of the damped Holt-winters model is obtained through the transformation of exponential smoothing method, as follows:

$$
y_{t+h}^{\prime}=\left[a_{t}+\left(\phi+\phi^{2}+\cdots+\phi^{h}\right) b_{t}\right] s_{t+h-m}
$$

where, a stands for intercept; $b$ is the trend; $s$ represents the seasonal factor of the damped model; $t$ represents the predicted time, and $t=1,2, \cdots, m ; h$ is the number of smooth phases, $h>0 ; m$ represents the cycle length, for example, 4 for the quarterly data and 12 for the monthly data. Since the experimental data interval in this paper is hour, 24 data are collected in a cycle, so $m$ is 24 .

The calculation formulas of $a, b$ and $s$ are as follows:

$$
\begin{gathered}
a_{t}=\alpha\left(y_{t} / s_{t-m}\right)+(1-\alpha)\left(a_{t-1}+\phi b_{t-1}\right) \\
b_{t}=\beta\left(a_{t}-a_{t-1}\right)+(1-\beta) \phi b_{t-1} \\
s_{t}=\gamma\left[y_{t} /\left(a_{t-1}-\phi b_{t-1}\right)\right]+(1-\gamma) s_{t-m}
\end{gathered}
$$

In the formula, $\alpha, \beta$ and $\gamma$ represent damped factors, also known as smoothing coefficient, and take values between 0 and 1 .

\section{The Empirical Analysis of Holt-Winters Model, Based on Slope Monitoring Data}

\subsection{Data Source}

The data of deep horizontal displacement of slope soil in this paper are derived from the SQL sever database of Sichuan Shengtuo detection Co., LTD., and the related slope engineering project is the ladder trough landslide monitoring project of Shuangma village, Shiguan township, Maoxian county. SQL-server 
database is a scalable, high-performance, computable for distributed servers relational data-base management system by Microsoft.

\subsection{Data Selection}

Due to the failure of data acquisition equipment and external environment, data acquisition at some time points is missing. Therefore, in order to reduce the prediction error, a continuous and stable period of data collection was selected for the prediction experiment.

Randomly selecting 500 - 650 data of a deep horizontal displacement monitoring point in the monitoring project, there are two reasons for choosing between 500 and 650. One is that if too little data is selected, the information in historical data cannot be fully extracted. On the contrary, the horizontal displacement with a long interval will have a small impact on the later prediction of horizontal displacement, resulting in unnecessary errors. The other is to look up many relevant literature about Holt-winters' model. The number of original sequences for empirical analysis of quarterly data $(m=4)$ is between 50 and 100 , and the number of monthly data $(m=12)$ is between 250 and 350 . Therefore, the number of original sequences for empirical analysis of time-scale data $(m=$ $24)$ is between 500 and 650 .

In the end, this paper selects the "X-axis" direction from 0 points on August 15, 2018 to 23 points on September 10, 2018, and all the integral point data from 0 points on August 21, 2018 to 23 points on September 10, 2018, and constructs the " $\mathrm{X}$ " data set and the "Y" data set, respectively, with a data quantity of 648 and 504, as shown in Figure 1 and Figure 2.

It can be seen from Figure 1 and Figure 2 that the series $\left\{X_{t}\right\}$ of " $X$ " data set and $\left\{Y_{t}\right\}$ of " $\mathrm{Y}$ " data set are obvious time series with deterministic trend and periodicity.

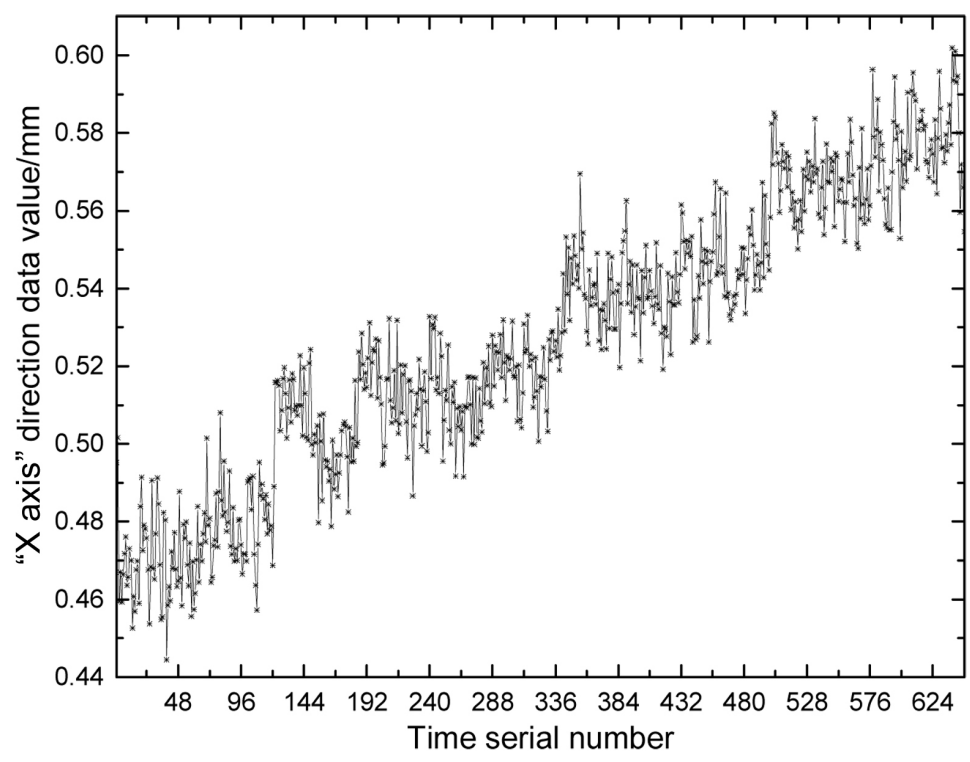

Figure 1. "X" data set sequence diagram. 


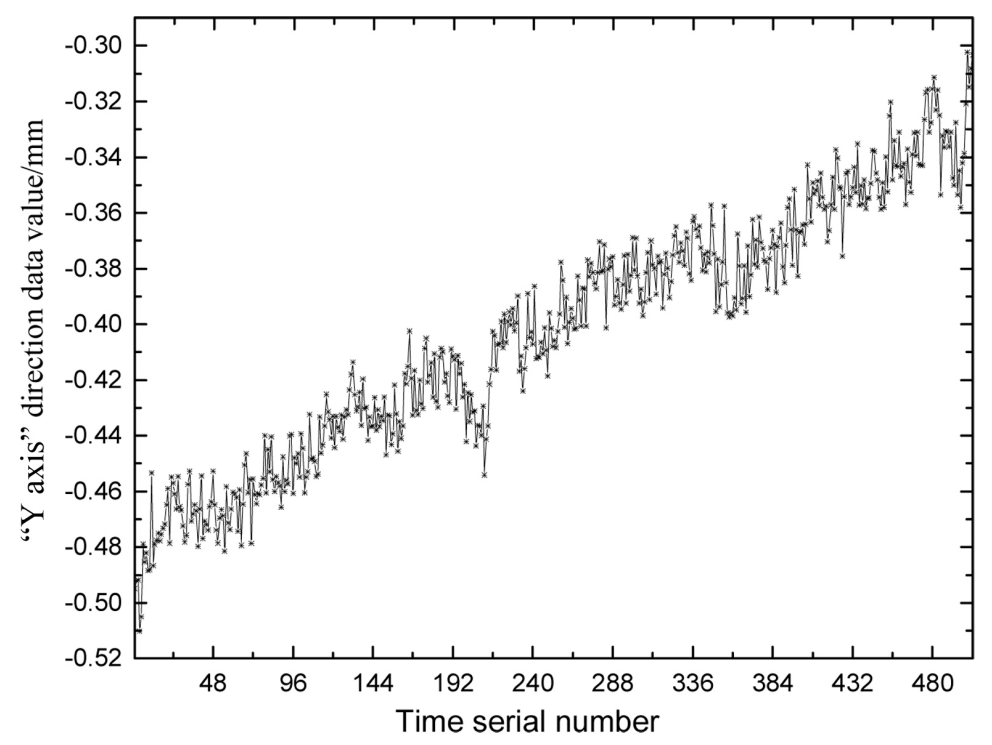

Figure 2. "Y" data set sequence diagram.

\subsection{Discrete Analysis}

In order to verify whether the data has the significance of predictive research, Origin-Pro data analysis software was used for the discrete analysis of data sets of "X" and "Y" to obtain the correlation coefficient and fitting degree values of data at adjacent moments, as shown in Table 1. According to the analysis results, the horizontal axis is the "monitoring data value at the current moment", and the vertical axis is "monitoring data value at the previous moment", and drawing the scatter diagram and fitting curve of, as shown in Figure 3 and Figure 4.

It can be seen from Table 1 that the correlation coefficients of the two data sets are $P_{r x}=0.944$ and $P_{r y}=0.9668$. Therefore, it can be judged that there is a strong correlation between adjacent data sets of "X" and "Y". It was also known that their fitting degree indexes were $R_{x}^{2}=0.8913$ and $R_{y}^{2}=0.9347$, both of which were close to 1 , suggesting that the reliability of the analysis results was also high. It can also be seen from Figure 3 and Figure 4 that the variation trend of discrete points is almost consistent with the fitted curve. Therefore, these two sets of data have the significance of predictive research and can be used for predictive experiments.

\subsection{Prediction and Result Analysis}

By using the WEKA data mining software forecast function, first of all, let $\alpha, \beta$ and $\gamma$ carry out equivalent iterative operation within the interval of $[0.01,1]$. The statistical prediction error $=\mid$ the actual value - the predicted value $\mid /$ actual value $\times$ $100 \%$. It is concluded that when $\alpha=0.03, \beta=0.03$ and $\gamma=0.03$, the average prediction error of "X" data set is the smallest, which is $1.69 \%$. When $\alpha=0.11, \beta=$ 0.11 and $\gamma=0.11$, the average prediction error of "Y" data set is the smallest, which is $3.16 \%$. 
Table 1. Table of results of discrete analysis.

\begin{tabular}{ccc}
\hline & "X" data set & "Y" data set \\
\hline Pearson's r & 0.9440 & 0.9668 \\
R-Square (COD) & 0.8913 & 0.9347 \\
\hline
\end{tabular}

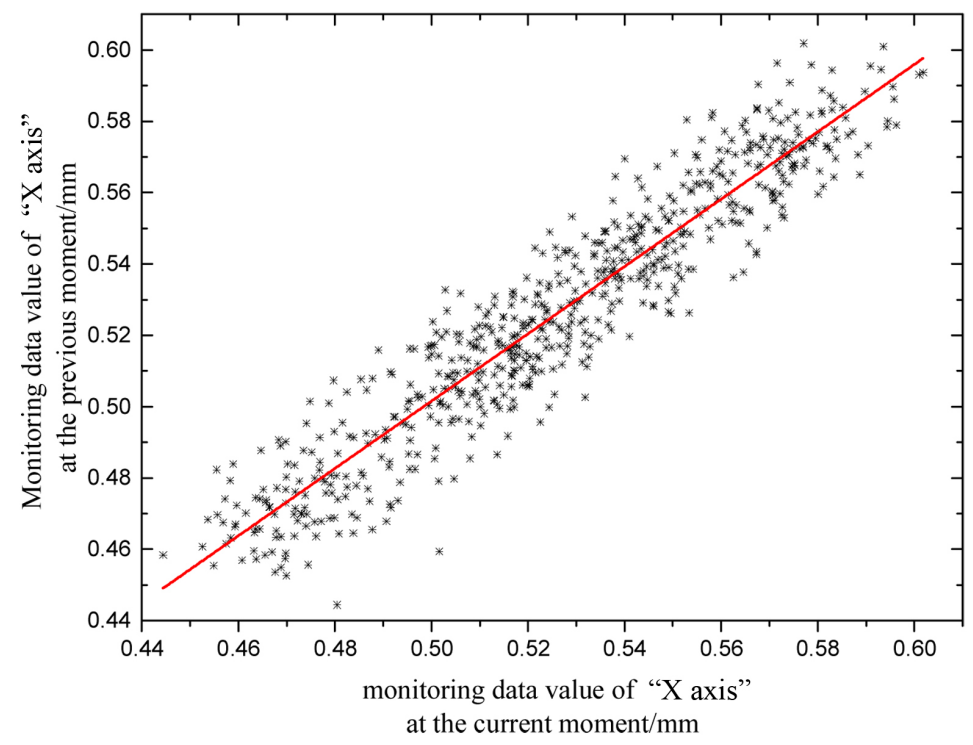

Figure 3. "X" data collection and distribution point diagram.

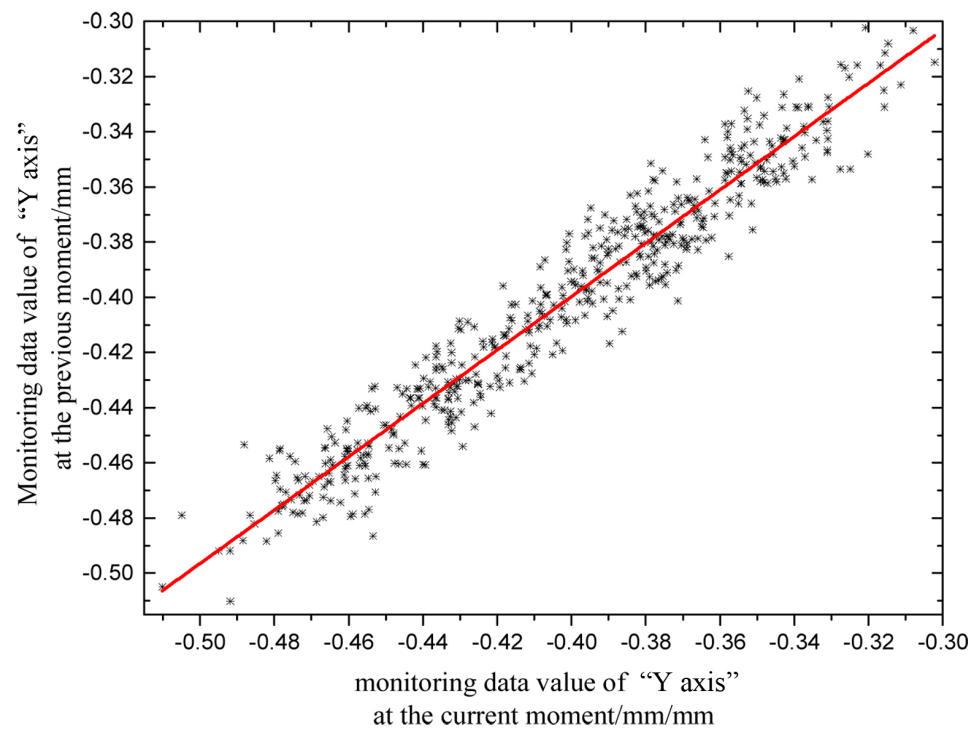

Figure 4. "Y" data collection and distribution point diagram.

The influence of smoothing coefficients $\alpha, \beta$ and $\gamma$ on the prediction error was analyzed again (the system default values of $\alpha, \beta$ and $\gamma$ were all 0.2 ). It can be found from Table 2 that, for the " $\mathrm{X}$ " data set, when $\beta$ is the default value, the changes of average prediction error is the most, about $0.75 \%$, so it can be inferred that the change of is most likely to improve the prediction accuracy. For the "Y" data set, when $\beta$ and $\gamma$ are the default values, the average prediction 
Table 2. Influence analysis of smoothing coefficient.

\begin{tabular}{ccc}
\hline Direction & Coefficient of size & Mean prediction error \\
\hline \multirow{2}{*}{$\alpha=0.2, \beta=0.03, \gamma=0.03$} & $1.53 \%$ \\
$\alpha=0.03, \beta=0.2, \gamma=0.03$ & $2.44 \%$ \\
$\alpha=0.03, \beta=0.03, \gamma=0.2$ & $1.84 \%$ \\
$\alpha=0.2, \beta=0.11, \gamma=0.11$ & $3.11 \%$ \\
"Y axis" & $\alpha=0.11, \beta=0.2, \gamma=0.11$ & $3.34 \%$ \\
& $\alpha=0.11, \beta=0.11, \gamma=0.2$ & $3.39 \%$ \\
\hline
\end{tabular}

error changes greatly, so it is possible to improve the prediction accuracy by changing $\beta$ and $\gamma$.

According to the above analysis results, for the " $\mathrm{X}$ " data set, it is determined that $\alpha$ and $\gamma$ are invariants and $\beta$ are variables. The analysis and prediction results show that when $\beta=0.04$, the minimum prediction error is about $1.68 \%$, as shown in Figure 5. For the data set of "Y", it is determined that $\alpha$ is the invariant and $\beta$ and $\gamma$ are the variables. It is concluded from the analysis of the prediction results that the smaller the value of $\gamma$ is, the more the value of $\beta$ increases, and the minimum prediction error is close to $3 \%$, as shown in Figure 6.

\subsubsection{Analysis of the Predicted Results of the "X" Data Set}

To sum up, when $\alpha=0.02, \beta=0.03$ and $\gamma=0.03$, the average prediction error of the direction of " $\mathrm{X}$ axis" is the smallest, which is $1.53 \%$. The predicted results are shown in Table 3.

In Table 3, the maximum and average prediction errors calculated by monitoring the actual values and the model predicted values were very small, which were $5.05 \%$ and $1.53 \%$, respectively, indicating that the short-term prediction accuracy of the damped Holt-winters model on the " $\mathrm{X}$ " data set was very high. According to the $95 \%$ confidence of the actual value, the upper and lower limits of the prediction were calculated and the prediction effect diagram of the prediction interval was drawn, as shown in Figure 7.

As can be seen from Figure 7, almost all the predicted points are within the predicted range (there is only one point slightly deviating from the predicted range, the blue point in the red rectangle in the figure). The predicted value and the actual value were consistent with each other. To sum up, the damped Holt-winters model may predict the deep horizontal displacement of slope soil of $\mathrm{X}$-axis direction.

\subsubsection{Analysis of the Predicted Results of the "Y" Data Set}

When $\alpha=0.11, \beta=0.23$ and $\gamma=0.05$, the average prediction error of the direction of "Y axis" is the smallest, which is $3.07 \%$. The predicted results are shown in Table 4.

In Table 4, the prediction error is obtained by calculating the actual monitoring value and the predicted model value. Although the maximum prediction error was $10.05 \%$, the average prediction error was $3.07 \%$, indicating that the 


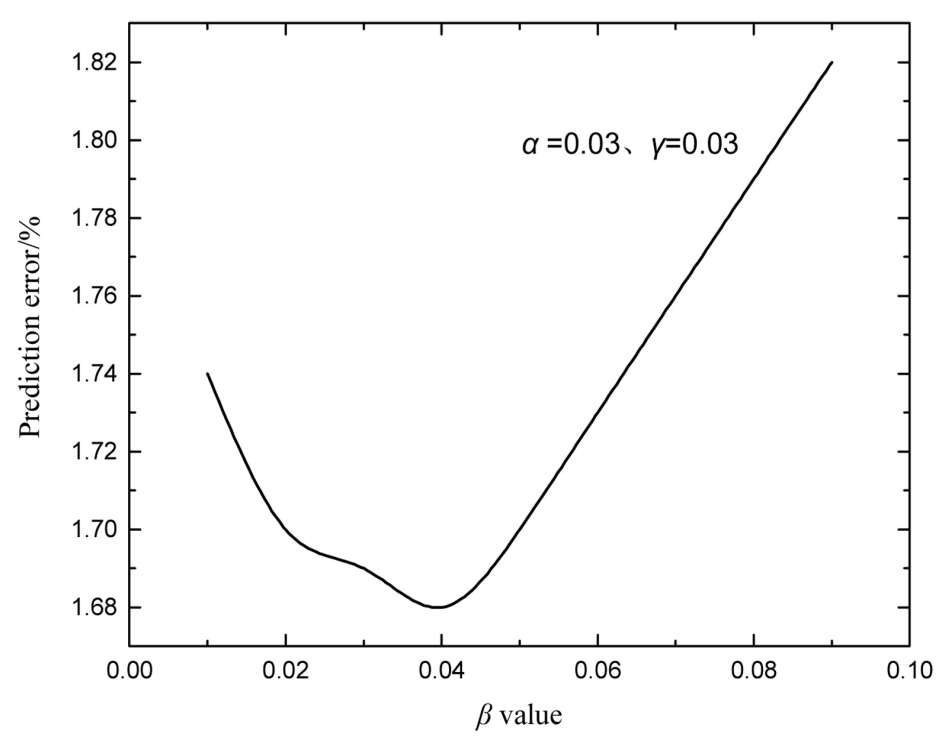

Figure 5. Changes in the prediction error of the " $\mathrm{X}$ " dataset.

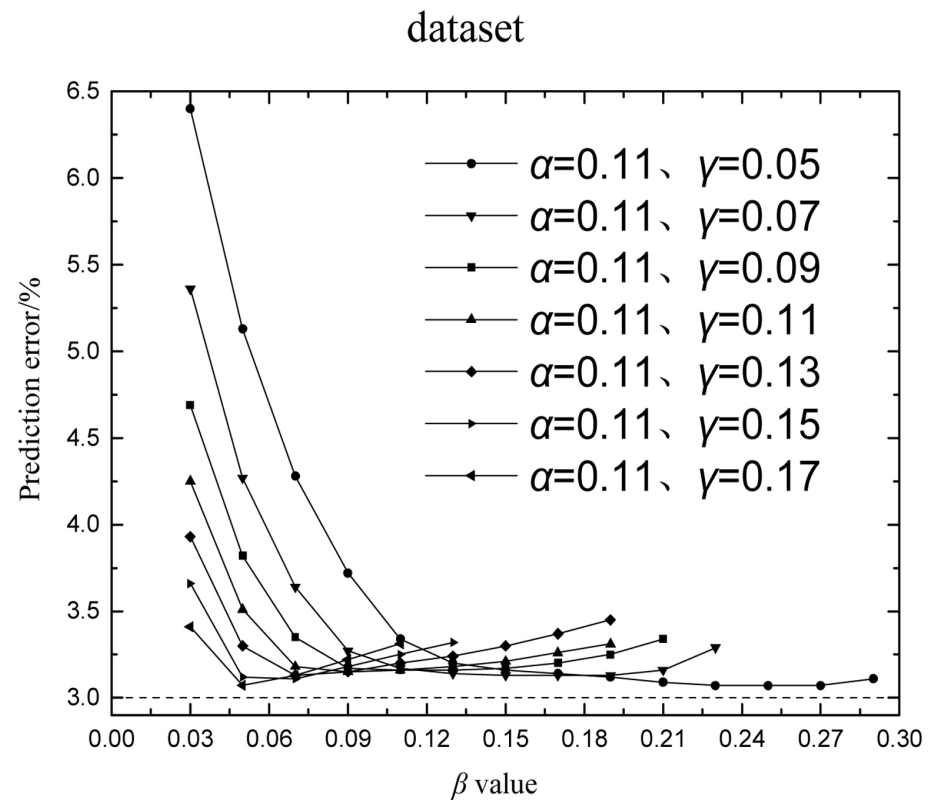

Figure 6. Changes in the prediction error of the "Y" dataset.

damped Holt-winters model had a high short-term prediction accuracy for "Y" dataset. According to the $95 \%$ confidence of the actual value, the upper and lower limits of the prediction were calculated and the prediction effect diagram of the prediction interval was drawn, as shown in Figure 8.

As can be seen from Figure 8, almost all the predicted points are within the predicted range (there is only three points slightly deviating from the predicted range, the blue point in the red rectangle in the figure). The predicted value and the actual value were consistent with each other. To sum up, the damped Holt-winters model may predict the deep horizontal displacement of slope soil of Y-axis direction. 


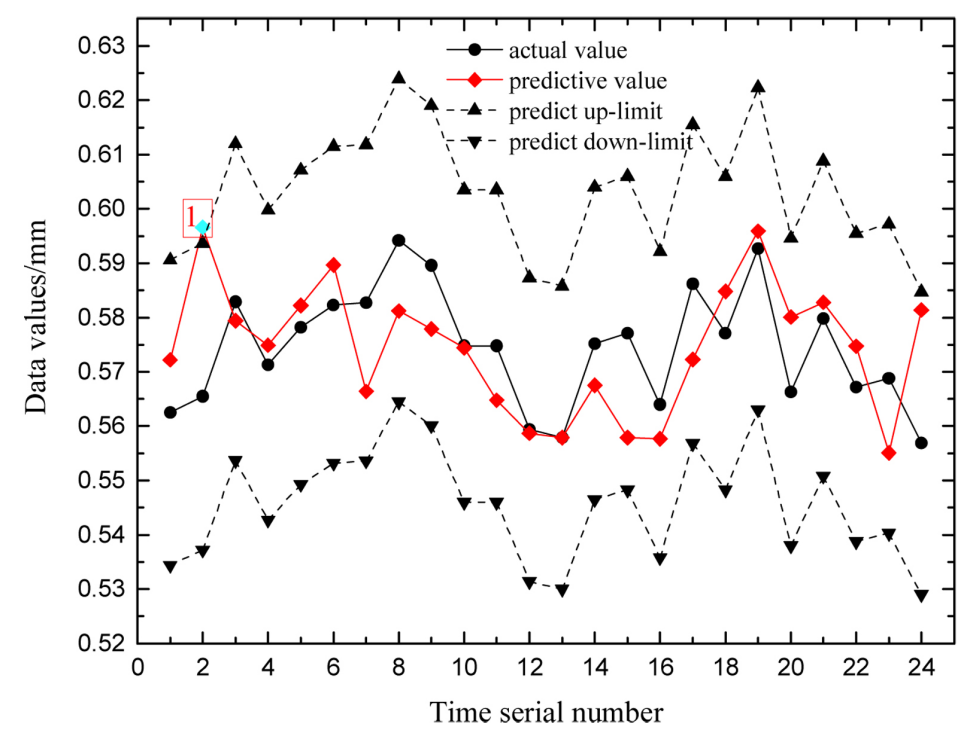

Figure 7. A predictive rendering of the " $\mathrm{X}$ " dataset.

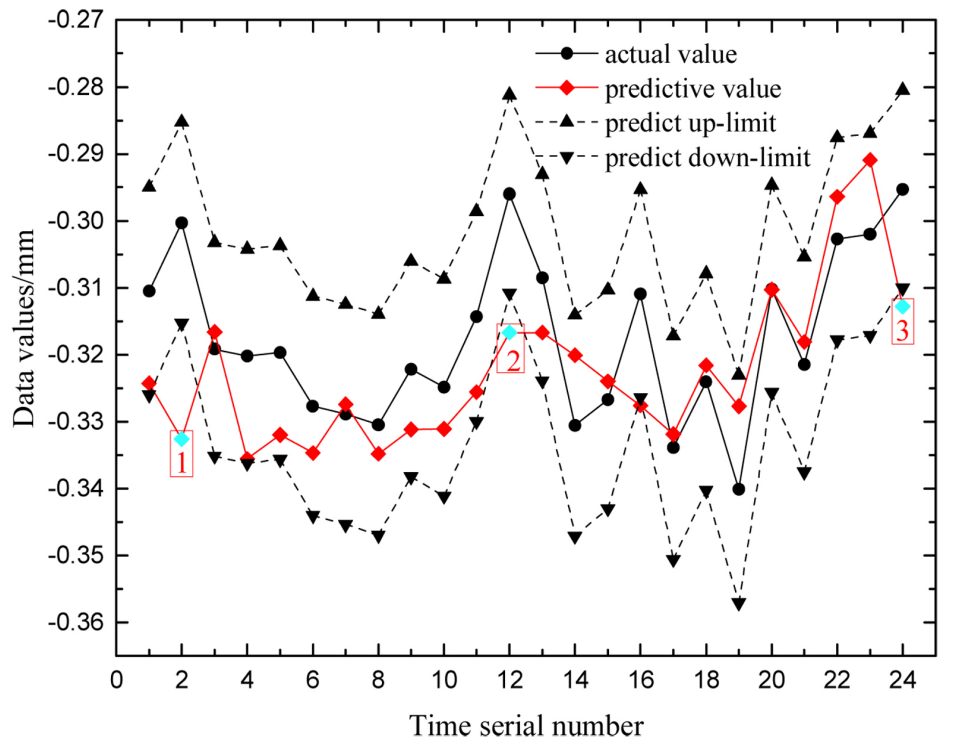

Figure 8. A predictive rendering of the " $\mathrm{Y}$ " dataset.

\subsection{Analysis of Experimental Results}

The predicted results of " $\mathrm{X}$ " and "Y" data sets were compared and analyzed, as shown in Figure 9 and Table 5.

As can be seen intuitively from Figure 10 and Table 5, although the prediction error of " $Y$ " dataset fluctuates greatly compared with that of " $\mathrm{X}$ " dataset, almost all of them are below 7\%. Their average prediction error is also very small, $1.53 \%$ and $3.07 \%$ respectively. Therefore, the damped Holt-winters model has a high accuracy in predicting the deep horizontal displacement of slope soil.

\section{Comparative Analysis of Traditional Models}

In order to further verify the reliability and feasibility of experimental results, 
Table 3. The prediction results of the "X-axis" direction.

\begin{tabular}{|c|c|c|c|}
\hline Time serial number & Actual value $/ 10^{-2} \mathrm{~mm}$ & Predictive value $/ 10^{-2} \mathrm{~mm}$ & Prediction error $/ \%$ \\
\hline 1 & 56.25 & 57.10 & 1.51 \\
\hline 2 & 56.55 & 59.40 & 5.05 \\
\hline 3 & 58.29 & 57.65 & 1.09 \\
\hline 4 & 57.13 & 57.53 & 0.70 \\
\hline 5 & 57.82 & 58.43 & 1.05 \\
\hline 6 & 58.23 & 58.87 & 1.09 \\
\hline 7 & 58.27 & 56.77 & 2.57 \\
\hline 8 & 59.42 & 57.99 & 2.40 \\
\hline 9 & 58.96 & 57.65 & 2.22 \\
\hline 10 & 57.48 & 57.48 & 0.00 \\
\hline 11 & 57.48 & 56.61 & 1.51 \\
\hline 12 & 55.94 & 56.22 & 0.51 \\
\hline 13 & 55.79 & 56.22 & 0.77 \\
\hline 14 & 57.52 & 56.82 & 1.22 \\
\hline 15 & 57.71 & 56.44 & 2.21 \\
\hline 16 & 56.40 & 56.25 & 0.26 \\
\hline 17 & 58.62 & 57.56 & 1.80 \\
\hline 18 & 57.71 & 58.45 & 1.28 \\
\hline 19 & 59.27 & 59.38 & 0.19 \\
\hline 20 & 56.63 & 57.83 & 2.11 \\
\hline 21 & 57.98 & 57.70 & 0.48 \\
\hline 22 & 56.72 & 57.25 & 0.94 \\
\hline 23 & 56.88 & 55.52 & 2.38 \\
\hline 24 & 55.69 & 57.51 & 3.27 \\
\hline
\end{tabular}

Note: the predicted time is "2018-09-10-2018-09-10 23". The actual value comes from the SQL sever data-base of Sichuan Shengtuo detection co., LTD.

Multilayer-Perceptron model and IBK model were used for prediction, and compared with the prediction results of the damped Holt-winters model. Curves were drawn, as shown in Figure 10 and Figure 11.

It can be intuitively seen from Figure 10 and Figure 11 that the curve variation trend of the predicted results of the three models is roughly the same. It can be concluded that both Multilayer-Perceptron model and IBK model can be used to predict the deep horizontal displacement of landslide soil mass, but the prediction accuracy is different. Therefore, their prediction errors are counted for further analysis, as shown in Table 6 and Table 7.

From the prediction error data can be seen in Table 6 and Table 7, in deep landslide soil horizontal displacement direction ("X", "Y" direction) monitoring data of prediction experiments, damped Holt-Winters model prediction error in turn is a maximum, minimum and average: $5.05 \%, 0 \%, 1.53 \%$ and $10.76 \%$, 
Table 4. The prediction results of the "Y-axis" direction.

\begin{tabular}{|c|c|c|c|}
\hline Time serial number & Actual value $/ 10^{-2} \mathrm{~mm}$ & Predictive value $/ 10^{-2} \mathrm{~mm}$ & Prediction error $/ \%$ \\
\hline 1 & -31.05 & -32.43 & 4.44 \\
\hline 2 & -30.03 & -33.26 & 10.76 \\
\hline 3 & -31.92 & -31.66 & 0.82 \\
\hline 4 & -32.02 & -33.56 & 4.80 \\
\hline 5 & -31.97 & -33.20 & 3.85 \\
\hline 6 & -32.77 & -33.47 & 2.15 \\
\hline 7 & -32.89 & -32.74 & 0.46 \\
\hline 8 & -33.05 & -33.48 & 1.31 \\
\hline 9 & -32.22 & -33.12 & 2.81 \\
\hline 10 & -32.49 & -33.11 & 1.90 \\
\hline 11 & -31.43 & -32.56 & 3.60 \\
\hline 12 & -29.60 & -31.67 & 6.99 \\
\hline 13 & -30.85 & -31.67 & 2.67 \\
\hline 14 & -33.06 & -32.01 & 3.18 \\
\hline 15 & -32.67 & -32.40 & 0.82 \\
\hline 16 & -31.09 & -32.76 & 5.37 \\
\hline 17 & -33.39 & -33.19 & 0.60 \\
\hline 18 & -32.41 & -32.16 & 0.78 \\
\hline 19 & -34.01 & -32.77 & 3.64 \\
\hline 20 & -31.02 & -31.03 & 0.04 \\
\hline 21 & -32.15 & -31.81 & 1.05 \\
\hline 22 & -30.27 & -29.64 & 2.08 \\
\hline 23 & -30.20 & -29.09 & 3.69 \\
\hline 24 & -29.53 & -31.28 & 5.94 \\
\hline
\end{tabular}

Note: the predicted time is "2018-09-10-2018-09-10 23". The actual value comes from the SQL sever data-base of Sichuan Shengtuo detection co., LTD.

Table 5. Statistical analysis of experimental results.

\begin{tabular}{lcc}
\hline & Avg/(\%) & $\mathrm{S}^{2}$ \\
\hline The prediction error of the "X" data set & 1.53 & $1.85 \mathrm{E}-04$ \\
The prediction error of the "Y" data set & 3.07 & $6.20 \mathrm{E}-04$ \\
\hline
\end{tabular}

Table 6. "X" data set prediction error statistics.

\begin{tabular}{cccc}
\hline & $\begin{array}{c}\text { damped Holt-Winters } \\
\text { model }\end{array}$ & $\begin{array}{c}\text { Multilayer-Perceptron } \\
\text { model }\end{array}$ & IBK model \\
\hline Max/\% & 5.05 & 6.68 & 4.85 \\
Min/\% & 0.00 & 0.23 & 0.24 \\
Avg/\% & 1.53 & 2.48 & 1.75 \\
\hline
\end{tabular}


Table 7. "Y" data set prediction error statistics.

\begin{tabular}{cccc}
\hline & $\begin{array}{c}\text { damped Holt-Winters } \\
\text { model }\end{array}$ & $\begin{array}{c}\text { Multilayer-Perceptron } \\
\text { model }\end{array}$ & IBK model \\
\hline Max/\% & 10.76 & 11.51 & 14.76 \\
Min/\% & 0.04 & 0.33 & 0.06 \\
Avg/\% & 3.07 & 3.97 & 4.21 \\
\hline
\end{tabular}

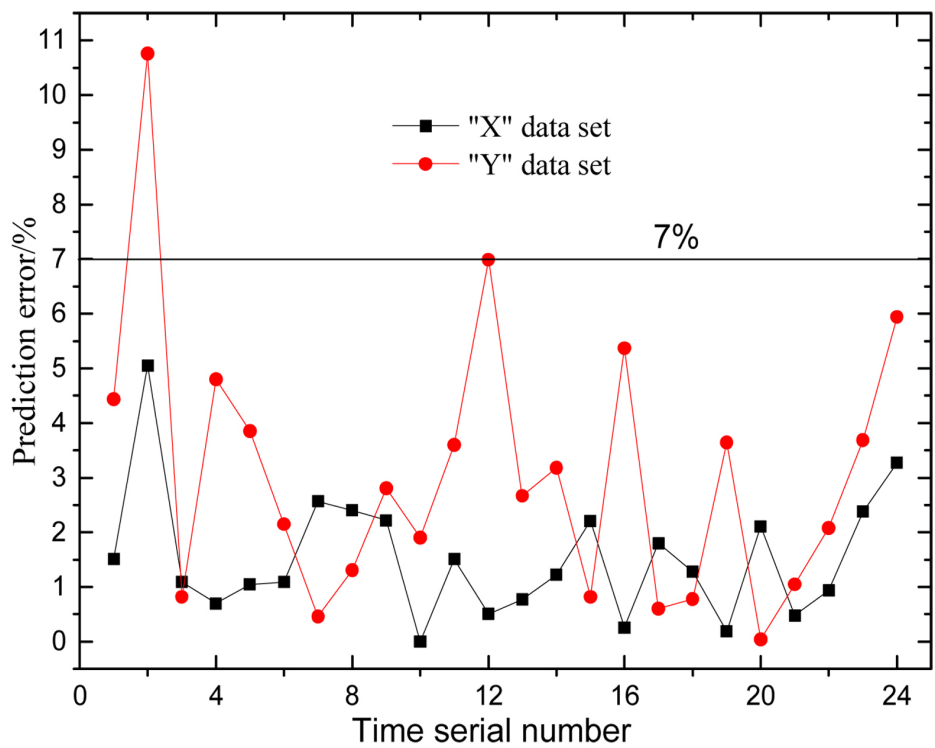

Figure 9. Change curve of prediction error $\mathrm{S}^{2}$.

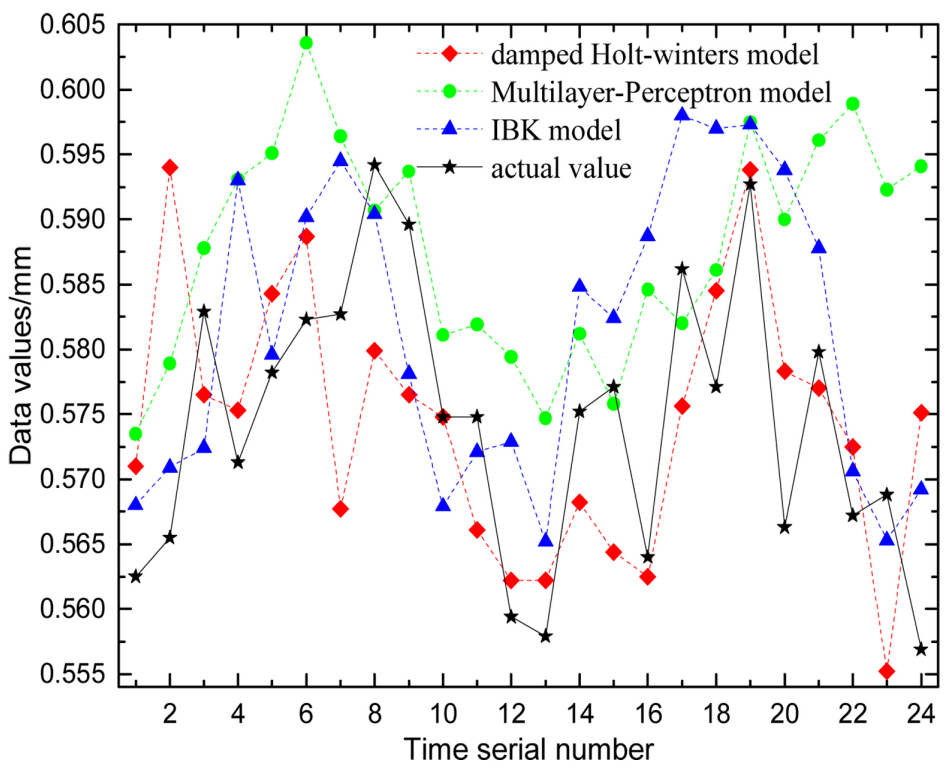

Figure 10. "X" data set prediction result.

$0.04 \%, 3.07 \%$, and all to optimal Multilayer Perceptron model, IBK model. The result show the damped Holt-winters model had the highest prediction accuracy and the best effect. 


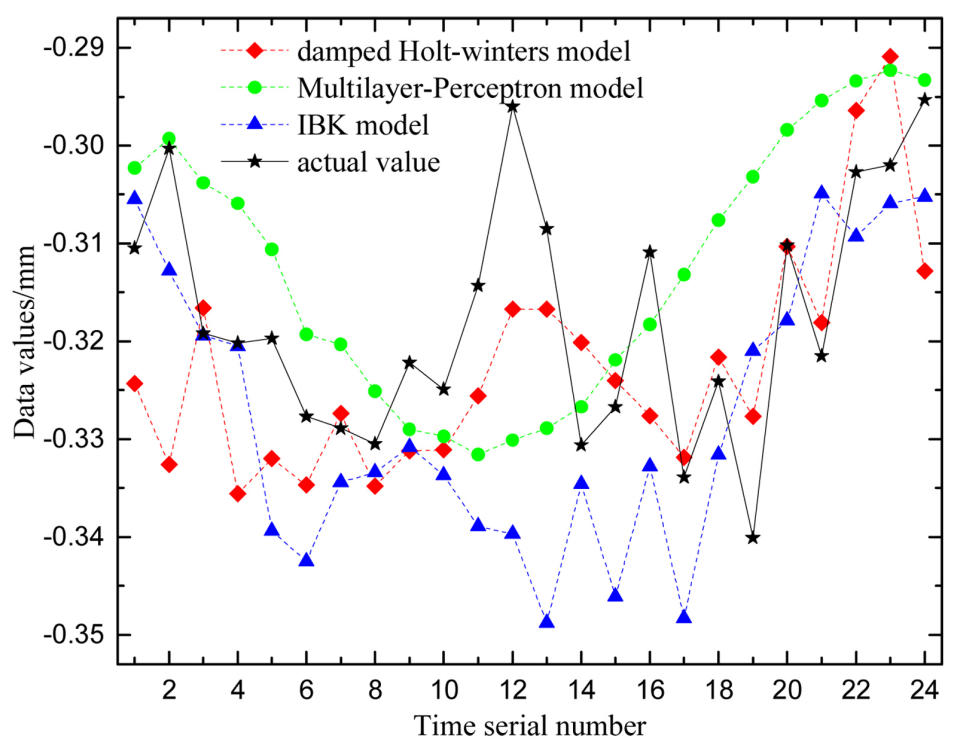

Figure 11. "Y" data set prediction result.

\section{A New Method of Slope Safety Management}

Combined with the above experimental results, a new implementation method of slope safety management is proposed, which is prediction method for deep horizontal displacement of soil, referred to as Pm-DHDS, as shown in Figure 12.

The working principle of this method is as follows: relying on the data set of actual horizontal displacement monitoring in the deep layer of slope soil, short-term prediction is conducted with the damped Holt-winters model, which is also known as the one-stage prediction. If the analysis results of the first-phase prediction are harmless to the slope deformation evolution, the first-phase prediction results will be put into the original time series for the second-phase prediction, and then the long-term prediction may generate cumulative errors. On the contrary, if the predicted analysis results in a certain period are harmful to the slope deformation evolution, the corresponding safety management measures should be implemented in advance, which also provides the basis for the slope stability analysis and better prevents the occurrence of slope safety accidents.

\section{Sum Up}

\subsection{Conclusion}

It is a challenging problem to predict the deep horizontal displacement of slope soil, but time series prediction has been considered as an effective method to predict the trend growth and seasonal fluctuation. Because the prediction of time series has a good short-term prediction effect, although this paper only carries out the short-term prediction of time series, in theory, the prediction period of time series can be extended by adding effective original data, and the short-term accuracy will also be improved. The empirical analysis in this paper also shows 


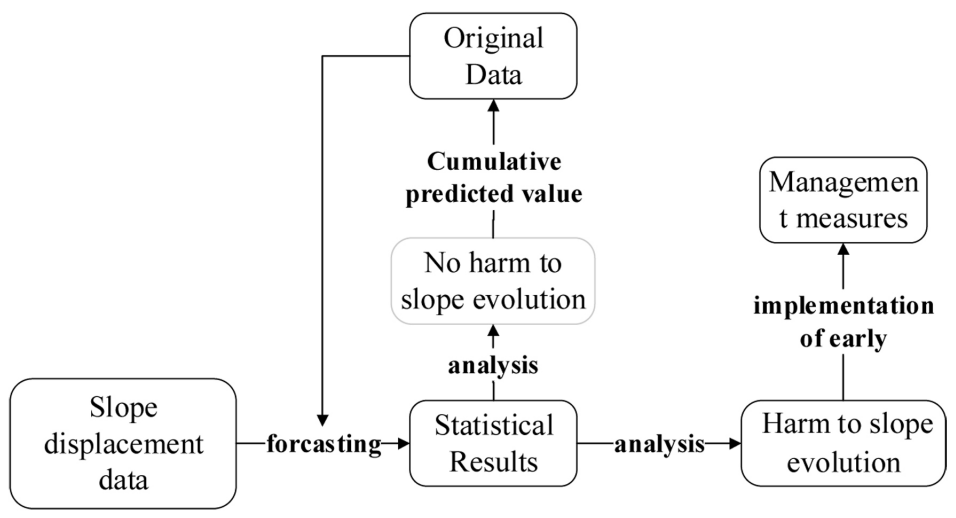

Figure 12. The workflow of the prediction method.

that the damped Holt-winters model is feasible as a short-term prediction model for deep horizontal displacement of slope soil, and its prediction accuracy is also the highest compared with the results of traditional methods. Thus, the application of damped Holt-winters model may also be of some reference value for this paper to grasp the implementation opportunity of slope safety management measures, analyze slope stability and prevent slope accidents.

Due to the limited time, this paper only makes an empirical analysis on the change of the actual data of the deep horizontal displacement of slope soil, and the difference in prediction accuracy is not significant, which indicates that the prediction of the same type of samples by the model has a strong stability. Therefore, for the prediction of different types of sample data, the conclusion may lack universality. In order to better connect the empirical analysis conclusion with the management work, the theoretical method of slope safety monitoring management is proposed, which is prediction method for deep horizontal displacement of soil, referred to as Pm-DHDS.

In conclusion, through the damped Holt-winters model, this paper used historical displacement values as serial data to make short-term prediction of the next 24 displacements, hoping to help slope safety managers to strengthen slope management and prevent slope accidents. At the same time, it can be considered to combine with other prediction methods to pay more attention to various factors such as slope structure factors, weather changes, engineering construction, etc. These uncertain factors often have important application value for the long-term trend of deep horizontal displacement of slope soil.

\subsection{Shortcomings and Prospects}

Shortcomings and prospects of this study are:

1) The damped Holt-winters model belongs to short-term prediction model, and its prediction effect will gradually deteriorate with the passage of time [16]. By analyzing the experimental results, it can be concluded that the experimental data set can be increased to make up for this deficiency. In future analysis experiments, more experimental data can be used for longer time prediction to verify this. 
2) Abnormal data may exist in the experimental data, leading to the reduced reliability of the predicted results. There are two reasons for the abnormal data: one is the data acquisition equipment problems, resulting in abnormal data; Second, the structure of the object itself is abnormal, so that the acquisition data variation. For prediction experiments, the second cause of data anomalies is a reasonable category. The data anomaly caused by the first reason is outside the controllable factors. In future prediction experiments, the algorithm model should be improved as much as possible. If abnormal data can be screened and screened in advance, the reliability of prediction results can be guaranteed.

\section{Fund Project}

Bridge nondestructive testing and engineering calculation key laboratory of Sichuan University Open Fund Project (2018QZY01).

\section{Conflicts of Interest}

The authors declare no conflicts of interest regarding the publication of this paper.

\section{References}

[1] Wen, Y. and Zhu, J. (2018) On the Prediction for the Slope Stability Based on the SAPSO-ELM. Journal of Safety and Environment, 18, 2146-2150.

[2] Zhang, H. and Luo, Y. (2012) Prediction Model for Slope Stability Based on Artificial Immune Algorithm. Journal of China Coal Society, 37, 911-917.

[3] Sharma, K.R., Mehta, S.B. and Jamwal, C.S. (2013) Cut Slope Stability Evaluation of NH-21 along Nalayan-Gambhrola Section, Bilaspur District, Himachal Pradesh, India. Natural Hazards, 66, 249-270. https://doi.org/10.1007/s11069-012-0469-X

[4] He, Y. (2015) Several Key Techniques Application Research of Slope-Oriented Deformation Monitoring-Take Gong Jiafang Slope Monitoring for Example. Chongqing Jiaotong University, Chongqing.

[5] Mei, F. and Shi, X. (2018) Study on Slope Treatment Scheme and Stability of Shallow Buried Tunnel. Yunnan Water Power, 34, 165-168.

[6] Xue, X., Zhang, W. and Liu, H. (2008) Evaluation of Slope Stability Based on SOFM Neural Network. Rock and Soil Mechanics, 29, 2236-2240.

[7] Pieraccini, M., Casagli, N., Luzi, G., et al. (2010) Landslide Monitoring by Ground-Based Radar Interferometry: A Field Test in Valdarno (Italy). International Journal of Remote Sensing, 24, 1385-1391. https://doi.org/10.1080/0143116021000044869

[8] Barla, G., Antolini, F., Barla, M., et al. (2010) Monitoring of the Beauregard Landslide (Aosta Valley, Italy) Using Advanced and Conventional Techniques. Engineering Geology, 116, 218-235. https://doi.org/10.1016/j.enggeo.2010.09.004

[9] Gischig, V., Amann, F., Moore, J.R., et al. (2010) Composite Rock Slope Kinematics at the Current Randa Instability, Switzerland, Based on Remote Sensing and $\mathrm{Nu}$ merical Modeling. Engineering Geology, 118, 37-53. https://doi.org/10.1016/j.enggeo.2010.11.006

[10] Ruan, Z., Wang, Y. and Yang, H. (2012) Monitoring and Analysis of Slope Gauge of Two Deep Cut Landslides in Bailong Road. Journal of China \& Foreign Highway, $32,45-51$. 
[11] Wang, Z. and Zhang, J. (2013) Land-Slides Monitoring Based on InSAR Technique. Journal of Geodesy and Geodynamics, 33, 87-91.

[12] Xu, M., Zhang, H., Li, H., et al. (2015) Open-Pit Slope Displacement Monitoring System Based on Measurement Robot. Science of Surveying and Mapping, No. 1, 38-41.

[13] Zhang, S., He, M., Luo, Y., et al. (2009) Modeling Virtual Dynamics for Pedestrian Microscopic Simulation. Journal of Transportation Systems Engineering and Information Technology, 9, 51-55.

[14] Gao, H. and Chen, H. (2004) The Model of Pedestrian Disturbing in Micro-Traffic Simulation. Technology \& Economy in Areas of Communications, No. 4, 58-60.

[15] Xi, G., Yue, J. and Zhou, B. (2012) The Forecasting Model of Ionospheric Delay Based on Holt-Winter. Bulletin of Surveying and Mapping, 9, 7-10.

[16] Yan, B., Chi, W., Fu, L., et al. (2017) Application of Arima Model and Holt-Winters Model in the Forecast of Discharge Number in Breast Surgery Department. Chinese Medical Record, 18, 50-54. 